Article

\title{
Three-component, one-pot synthesis of benzo[f]indenoquinoline derivatives catalyzed by poly(4-vinylpyridinium) hydrogen sulfate
}

\author{
Nader Ghaffari Khaligh a,b,* \\ aDepartment of Chemistry, Qaemshahr branch, Islamic Azad University, Qaemshahr 47651-61964, Iran \\ ${ }^{\mathrm{b}}$ House Research of Professor Reza, Education Guilan, Rasht, District 1, 41569-17139, Iran
}

A R T I C L E I N F O

Article history:

Received 18 October 2013

Accepted 14 November 2013

Published 20 April 2014

\section{Keywords:}

Benzoquinoline

Hetereogeneous catalyst

2-Naphthylamine

Indane-1,3-dione

Three-component reaction

One-pot condensation

\begin{abstract}
A B S T R A C T
7-Aryl-8H-benzo[f]indeno[2,1- $b]$ quinoline-8-one derivatives were synthesized by means of a one-pot condensation of 2-naphthylamine, aromatic aldehydes, and indane-1,3-dione in ethanol under refluxing conditions in the presence of poly(4-vinylpyridinium) hydrogen sulfate, a solid acid catalyst. This method has the advantages of high yield, clean reaction, simple methodology, and short reaction time. The catalyst could be recycled and reused four times without significant loss of activity. The structure of the novel compounds was confirmed by IR, ${ }^{1} \mathrm{H}$ NMR, and ${ }^{13} \mathrm{C}$ NMR spectroscopy and elemental analysis.
\end{abstract}

(C) 2014, Dalian Institute of Chemical Physics, Chinese Academy of Sciences. Published by Elsevier B.V. All rights reserved.

\section{Introduction}

Quinoline and its derivatives are an important class of $\mathrm{N}$-containing heterocycles; they are present in a wide variety of naturally occurring alkaloids with pharmacological properties [1], and they are useful for dyes and intermediates in organic synthesis [2-9]. Quinoline compounds possess a broad spectrum of biological activity, such as antiasthmatic, anti-inflammatory, antimalarial, anticancer, and anthelmintic activity [10-19]. In addition, these compounds are well-known ligands for the preparation of organic light-emitting diode phosphorescent complexes [20-27], and they are valuable synthons for the preparation of nano- and mesostructures with enhanced electronic and photonic properties [28-31]. Benzoquinolines are used as metal ligands [32-37], and they occur in numerous chemical species with biological and pharmacological activity [38-41]. Benzo[h]quinolines have been widely used as rigid substrates to develop $\mathrm{C}-\mathrm{H}$ functionalization methodology [42] and as G-quadruplex binding agents [43-46]. Quinolines and benzoquinolines bearing five- or six-membered rings in a linear arrangement are known to possess good pharmacological and antimicrobial activity [47-55] and are also used as intermediates in the dye industry [56]. For example, indenoquinoline derivatives serve as antimalarial [57-68] and anticancer drugs [69-73]. Because of the utility of these compounds, numerous synthetic strategies have been developed for the preparation of substituted quinolines [74-80]. Most of the strategies involve the formation of the pyridine ring from a substituted naphthalene precursor [81-83].

In our laboratory, we have concentrated on developing quinoline and benzoquinoline compounds containing an indandione moiety. For example, we recently described an efficient one-pot synthesis of 12 -aryl-12H-indeno[1,2- $b]$ naphtho [2,3-e]pyran-6,11,13-trione derivatives in the presence of

\footnotetext{
* Corresponding author. Tel: +98-2166431738; Fax: +98-2166934046; E-mail: ngkhaligh@guilan.ac.ir DOI: 10.1016/S1872-2067(12)60752-9 | http://www.sciencedirect.com/science/journal/18722067 | Chin. J. Catal., Vol. 35, No. 4, April 2014
} 
poly(4-vinylpyidinium) hydrogen sulfate under solvent-free conditions [84]. Mamaghani et al. [85] reported the synthesis of benzo[ $h]$ indeno[2,1- $b]$ quinoline-8-one under solvent-free conditions with ultrasound irradiation. To the best of our knowledge, there have been only a few other reports on the synthesis of benzo[ $h]$ indeno[2,1- $b]$ quinoline-8-one derivatives [86-89]. Therefore, herein, we describe the optimization of reaction conditions for the synthesis of 7-aryl-8H-benzo [f] indeno $[2,1-b]$ quinoline-8-one derivatives, which may be useful as dyes or luminescent materials or may exhibit pharmaceutical activity.

The use of solid acid catalysts offers unique advantages in organic synthesis, including operational simplicity, environmental compatibility, nontoxicity, low cost, and ease of isolation. As economical and ecologically benign catalysts, their applications have attracted much attention in chemistry and industry [90-98]. However, they also have some disadvantages that reduce their utility [99]. Although zeolites demonstrate high reactivity, their reactions typically give a variety of undesired by-products owing to the higher temperatures employed [100] Ion exchange resins are limited in application because they are thermally unstable above $120{ }^{\circ} \mathrm{C}$ in their acidic form [101].

\section{Experimental}

\subsection{General}

Unless otherwise stated, all chemicals were purchased from Fluka AG, Merck, or Synthetic Chemicals. Poly(4-vinylpyridine) cross-linked with $2 \%$ divinylbenzene was purchased from Fluka AG. Reactions were monitored and product purity was determined by TLC or by GC-MS on an Agilent GC-Mass-6890 instrument operating at $70 \mathrm{eV}$. IR and FTIR spectra in the range of 4000-400 $\mathrm{cm}^{-1}$ were obtained with a Perkin-Elmer 781 spectrometer and a Bruker Equinox 55 spectrometer, respectively, using $\mathrm{KBr}$ pellets for solids or neat for liquid samples. All ${ }^{1} \mathrm{H}$ NMR and ${ }^{13} \mathrm{C}$ NMR spectra were recorded with Bruker Avance 400 and $100 \mathrm{MHz}$ instrument, respectively. ${ }^{13} \mathrm{C}$ NMR data were collected on Bruker Advance $100 \mathrm{MHz}$ instrument. All chemical shifts are quoted in parts per million (ppm) relative to TMS in a deuterated solvent. Microanalyses were performed on a Perkin-Elmer 240-B microanalyzer. Melting points were recorded on a Büchi B-545 apparatus in open capillary tubes.

\subsection{Typical procedure for the preparation of 7-aryl-8H-benzo[f] indeno [2,1-b] quinoline-8-ones 4}

Poly(4-vinylpyridinium) hydrogen sulfate [102] $\left(\mathrm{P}(4-\mathrm{VPH}) \mathrm{HSO}_{4}, 20 \mathrm{mg}\right)$ was added to a mixture of 2-naphthylamine 1 (1.1 mmol), an aromatic aldehyde 2 (1 $\mathrm{mmol})$, and indane-1,3-dione 3 (1 mmol) in ethanol $(5 \mathrm{~mL})$, and the mixture was stirred for $2 \mathrm{~h}$ under refluxing conditions (Table 1). After completion of the reaction, the solvent was evaporated, and the residue was diluted with $\mathrm{CH}_{2} \mathrm{Cl}_{2}(5 \mathrm{~mL})$; the catalyst was recovered by filtration, washed with ethyl ether ( 5
$\mathrm{mL}$ ) and acetone (5 mL), and dried at ambient temperature. The recovered catalyst was reused for the next run. The solvent was evaporated from the filtrate at reduced pressure, and the solid residue was purified by silica gel column chromatography.

\subsection{Activation of $\mathrm{P}(4-\mathrm{VPH}) \mathrm{HSO}_{4}$}

Used catalyst collected from the experiments was washed successively with ethyl ether and acetone, dried under vacuum at room temperature, and then stirred with sulfuric acid to restore its activity.

\subsection{Analytical data for the products}

7-(Phenyl)-8H-benzo[f]indeno-[1,2- $b]$ quinoline-8-one (4a). Pale yellow needles; mp: $243-245{ }^{\circ} \mathrm{C}$. IR (KBr): $v_{\max }=1714$, 1621, 1570, 1510, 1482, $1080 \mathrm{~cm}^{-1} .{ }^{1} \mathrm{H}$ NMR (400 MHz, $\mathrm{CDCl}_{3}$ ): $\delta 9.54(\mathrm{~d}, J=8.0 \mathrm{~Hz}, 1 \mathrm{H}), 8.23(\mathrm{~d}, J=7.7 \mathrm{~Hz}, 1 \mathrm{H}), 7.90$ (d, $J=7.7$ $\mathrm{Hz}, 1 \mathrm{H}), 7.85-7.75(\mathrm{~m}, 5 \mathrm{H}), 7.64-7.61$ (m, 4H), 7.51-7.46 (m, 3H). ${ }^{13} \mathrm{C}$ NMR $\left(100 \mathrm{MHz}, \mathrm{CDCl}_{3}\right): \delta 191.4,162.5,149.7,148.1$, 144.0, 137.2, 135.5, 135.1, 133.6, 132.1, 131.5, 130.1, 129.5, 129.3, 128.6, 128.3, 127.5, 126.1, 125.4, 124.5, 124.1, 123.8, 121.7. Anal. Calcd for $\mathrm{C}_{26} \mathrm{H}_{15} \mathrm{NO}$ : C 87.39\%, H 4.20\%, N 3.92\%. Found: C 87.31\%, H 4.22\%, N 3.88\%.

7-(4-Chlorophenyl)-8H-benzo[f]indeno-[1,2-b]quinoline-8one (4b). Pale yellow needles; mp: $280-282^{\circ} \mathrm{C}$. IR (KBr): $v_{\max }=$ 3072, 1700, 1602, 1591, 1574, 1556, 1488, 1083, 842, 815, 750, $741 \mathrm{~cm}^{-1} .{ }^{1} \mathrm{H}$ NMR (400 MHz, $\mathrm{CDCl}_{3}$ ): $\delta 9.51(\mathrm{~d}, J=8.0 \mathrm{~Hz}$, $1 \mathrm{H}), 8.21(\mathrm{~d}, J=7.6 \mathrm{~Hz}, 1 \mathrm{H}), 7.90(\mathrm{~d}, J=7.6 \mathrm{~Hz}, 1 \mathrm{H}), 7.81-7.72$ (m, 5H), 7.60-7.54 (m, 3H), $7.52(\mathrm{t}, J=7.6 \mathrm{~Hz}, 1 \mathrm{H}), 7.41(\mathrm{~d}, J=$ $8.0 \mathrm{~Hz}, 2 \mathrm{H}) .{ }^{13} \mathrm{C}$ NMR (100 MHz, $\left.\mathrm{CDCl}_{3}\right): \delta 192.2,163.9,152.1$, 148.2, 146.1, 139.4, 137.5, 137.3, 136.8, 134.0, 133.9, 133.7, $133.4,131.7,131.2,130.5,130.1,129.6,128.1,127.2,126.3$, 126.0, 125.3, 124.1. Anal. Calcd for $\mathrm{C}_{26} \mathrm{H}_{14} \mathrm{ClNO}$ : C 79.69\%, H $3.57 \%$, N 3.57\%. Found: C 79.65\%, H 3.55\%, N 3.50\%.

7-(2-Chlorophenyl)-8H-benzo[f]indeno-[1,2- $b]$ quinoline-8one (4c). Yellow needles; mp: $300-302{ }^{\circ} \mathrm{C}$. IR (KBr): $v_{\max }=$ $1714,1600,1582,1573,1471,1045,832,808,792,755,734$ $\mathrm{cm}^{-1} .{ }^{1} \mathrm{H}$ NMR (400 MHz, $\left.\mathrm{CDCl}_{3}\right): \delta 9.51(\mathrm{~d}, J=8.0 \mathrm{~Hz}, 1 \mathrm{H}), 8.05$ (d, $J=7.4 \mathrm{~Hz}, 1 \mathrm{H}), 7.76(\mathrm{~d}, J=7.7 \mathrm{~Hz}, 1 \mathrm{H}), 7.65-7.51(\mathrm{~m}, 5 \mathrm{H})$, 7.46 (d, $J=8.0 \mathrm{~Hz}, 1 \mathrm{H}), 7.41-7.33(\mathrm{~m}, 3 \mathrm{H}), 7.22-7.19(\mathrm{~m}, 2 \mathrm{H})$. ${ }^{13} \mathrm{C}$ NMR (100 MHz, $\mathrm{CDCl}_{3} / \mathrm{DMSO}^{\left.-\mathrm{d}_{6}\right):} \delta$ 191.1, 162.3, 151.5, 149.7, 144.2, 144.1, 137.1, 135.5, 134.8, 133.2, 133.1, 132.0, $131.5,131.0,130.4,129.8,129.5,128.6,128.0,127.8,127.2$, 126.1, 125.0, 124.3, 124.0, 121.9. Anal. Calcd for $\mathrm{C}_{26} \mathrm{H}_{14} \mathrm{ClNO}$ : C 79.69\%, H 3.57\%, N 3.57\%. Found: C 79.64\%, H 3.51\%, N $3.53 \%$.

7-(3-Chlorophenyl)-8H-benzo[f]indeno-[1,2- $b]$ quinoline-8one (4d). Yellow solid; mp: $290-292{ }^{\circ} \mathrm{C}$. IR (KBr): $v_{\max }=3065$, 1711, 1604, 1573, 1553, 1471, 1045, 862, 842, 814, 805, 751, $724 \mathrm{~cm}^{-1}$; ${ }^{1} \mathrm{H}$ NMR (400 MHz, $\left.\mathrm{CDCl}_{3}\right): \delta 9.44(\mathrm{~d}, J=8.0 \mathrm{~Hz}, 1 \mathrm{H})$, $8.15(\mathrm{~d}, J=7.4 \mathrm{~Hz}, 1 \mathrm{H}), 7.85(\mathrm{~d}, J=7.8 \mathrm{~Hz}, 1 \mathrm{H}), 7.73-7.61(\mathrm{~m}$, 5H), 7.50-7.41 (m, 5H), $7.28(\mathrm{~m}, 1 \mathrm{H}) .{ }^{13} \mathrm{C}$ NMR $(100 \mathrm{MHz}$, $\left.\mathrm{CDCl}_{3}\right): \delta 190.8,162.1,150.3,144.1,142.2,138.0,135.0,135.2$, $135.0,133.5,131.2,131.0,130.8,130.6,129.5,129.0,128.6$, 128.2, 127.6, 127.4, 126.0, 125.0, 124.2, 124.0, 123.4, 121.9. Anal. Calcd for $\mathrm{C}_{26} \mathrm{H}_{14} \mathrm{ClNO}$ : C $79.69 \%$, H $3.57 \%$, N $3.57 \%$. 
Found: C 79.65\%, H 3.58\%, N 3.52\%.

7-(2,4-Dichlorophenyl)-8H-benzo[f]indeno-[1,2- $b]$ quinoline -8-one (4e). Yellow solid; mp: $256-258{ }^{\circ} \mathrm{C}$. IR (KBr): $v_{\max }=$ 3085, 1715, 1605, 1570, 1470, 1100, 1065, 852, 840, 812, 798, $772,755 \mathrm{~cm}^{-1}$. ${ }^{1} \mathrm{H}$ NMR (400 MHz, $\left.\mathrm{CDCl}_{3}\right): \delta 9.52(\mathrm{~d}, J=8.1 \mathrm{~Hz}$, $1 \mathrm{H}), 8.22$ (d, $J=7.4 \mathrm{~Hz}, 1 \mathrm{H}), 7.92$ (d, $J=7.7 \mathrm{~Hz}, 1 \mathrm{H}), 7.83-7.69$ $(\mathrm{m}, 5 \mathrm{H}), 7.70(\mathrm{~d}, J=1.90 \mathrm{~Hz}, 1 \mathrm{H}), 7.52-7.50(\mathrm{~m}, 2 \mathrm{H}), 7.38(\mathrm{~d}, J=$ $8.5 \mathrm{~Hz}, 1 \mathrm{H}), 7.34$ (d, J = 8.5 Hz, 1H). ${ }^{13} \mathrm{C} \mathrm{NMR}\left(100 \mathrm{MHz}, \mathrm{CDCl}_{3}\right)$ : $\delta$ 191.0, 162.1, 150.0, 144.3, 143.0, 137.3, 136.0, 135.8, 135.0, $134.5,132.1,132.0,131.9,131.8,130.1,129.9,128.8,128.3$, 127.8, 127.7, 126.2, 125.1, 124.4, 124.3, 123.7, 122.0. Anal. Calcd for $\mathrm{C}_{26} \mathrm{H}_{13} \mathrm{Cl}_{2} \mathrm{NO}$ : C $73.24 \%$, H 3.05\%, N 3.28\%. Found: C 73.21\%, H 3.08\%, N 3.21\%.

7-(4-Bromophenyl)-8H-benzo[f]indeno-[1,2- $b]$ quinoline-8one (4f). Yellow solid; mp: $278-280{ }^{\circ} \mathrm{C}$. IR (KBr): $v_{\max }=3060$, 1705, 1600, 1570, 1550, 1480, 1010, 995, 840, 810, 760, 740 $\mathrm{cm}^{-1} .{ }^{1} \mathrm{H}$ NMR (400 MHz, $\mathrm{CDCl}_{3}$ ): $\delta 9.47(\mathrm{~d}, J=8.0 \mathrm{~Hz}, 1 \mathrm{H}), 8.17$ $(\mathrm{d}, J=7.7 \mathrm{~Hz}, 1 \mathrm{H}), 7.87(\mathrm{~d}, J=7.8 \mathrm{~Hz}, 1 \mathrm{H}), 7.78-7.66(\mathrm{~m}, 7 \mathrm{H})$, $7.51(\mathrm{~d}, J=9.0 \mathrm{~Hz}, 1 \mathrm{H}), 7.46(\mathrm{t}, J=7.5 \mathrm{~Hz}, 1 \mathrm{H}), 7.32(\mathrm{~d}, J=8.2 \mathrm{~Hz}$, 2H). ${ }^{13} \mathrm{C}$ NMR $\left(100 \mathrm{MHz}, \mathrm{CDCl}_{3}\right): \delta 190.8,162.1,149.8,146.4$, $144.0,137.3,135.7,134.9,132.6,132.0,131.8,131.7,131.6$, 129.8, 128.4, 128.0, 127.5, 126.1, 125.2, 124.2, 124.1, 123.7, 123.3, 122.1. Anal. Calcd for $\mathrm{C}_{26} \mathrm{H}_{14} \mathrm{BrNO}$ : C 71.56\%, H 3.21\%, N $3.21 \%$. Found: C 71.54\%, H 3.24\%, N 3.18\%.

7-(4-Fluorophenyl)-8H-benzo[f]indeno-[1,2-b]quinoline-8one (4g). Yellow solid; mp: $259-260{ }^{\circ} \mathrm{C}$. IR (KBr): $v_{\max }=1709$, $1603,1572,1504,1155 \mathrm{~cm}^{-1}$. ${ }^{1} \mathrm{H}$ NMR (400 MHz, $\left.\mathrm{CDCl}_{3}\right): \delta 9.52$ (d, $J=8.1 \mathrm{~Hz}, 1 \mathrm{H}), 8.21(\mathrm{~d}, J=8.0 \mathrm{~Hz}, 1 \mathrm{H}), 7.89(\mathrm{~d}, J=7.2 \mathrm{~Hz} 1 \mathrm{H})$, 7.80-7.69 (m, 5H), 7.57 (d, $J=9.1 \mathrm{~Hz}, 1 \mathrm{H}), 7.50-7.46(\mathrm{~m}, 3 \mathrm{H})$, $7.20(\mathrm{~d}, J=8.6 \mathrm{~Hz}, 2 \mathrm{H}) .{ }^{13} \mathrm{C}$ NMR $\left(100 \mathrm{MHz}, \mathrm{CDCl}_{3}\right): \delta 191.2$, 162.2, 149.7, 146.7, 144.1, 137.3, 135.5, 134.8, 132.1, 131.9, 131.6, 129.8, 129.5, 128.3, 128.0, 127.6, 126.2, 125.5, 124.2, 123.8, 123.6, 122.0, 115.8. Anal. Calcd for $\mathrm{C}_{26} \mathrm{H}_{14} \mathrm{FNO}$ : C $83.20 \%$, H 3.73\%, N 3.73\%. Found: C 83.17\%, H 3.74\%, N 3.69\%.

7-(4-Methylphenyl)-8H-benzo[f]indeno-[1,2- $b]$ quinoline-8one (4h). Yellow solid; mp: $275-277^{\circ} \mathrm{C}$. IR (KBr): $v_{\max }=1707$, 1608, 1572, 1502, $1344 \mathrm{~cm}^{-1}$. ${ }^{1} \mathrm{H}$ NMR (400 MHz, $\left.\mathrm{CDCl}_{3}\right): \delta 9.52$ (d, $J=8.0 \mathrm{~Hz}, 1 \mathrm{H}), 8.20$ (d, $J=7.2 \mathrm{~Hz}, 1 \mathrm{H}), 7.88$ (d, $J=7.6 \mathrm{~Hz}$, $1 \mathrm{H}), 7.80-7.61(\mathrm{~m}, 5 \mathrm{H}), 7.47(\mathrm{t}, J=7.40 \mathrm{~Hz}, 1 \mathrm{H}), 7.40(\mathrm{~d}, J=8.0$ $\mathrm{Hz}, 2 \mathrm{H}), 7.37$ (d, J = 8.0 Hz, 2H), 2.54 (s, 3H). ${ }^{13} \mathrm{C} \mathrm{NMR}(100 \mathrm{MHz}$, $\left.\mathrm{CDCl}_{3}\right): \delta 191.1,162.4,149.4,148.1,144.0,139.2,137.4,135.5$, $134.9,132.0,131.5,130.8,130.1,129.6,129.3,128.1,128.0$, 127.6, 126.2, 125.7, 124.7, 124.1, 123.6, 121.9, 22.0. Anal. Calcd for $\mathrm{C}_{27} \mathrm{H}_{17} \mathrm{NO}$ : C 87.33\%, H 4.58\%, N 3.77\%. Found: C 83.27\%, H $4.55 \%$, N $3.74 \%$.
7-(4-Methoxylphenyl)-8H-benzo[f]indeno-[1,2-b]quinoline8-one (4i). Yellow solid; mp: $298-300{ }^{\circ} \mathrm{C}$. IR (KBr): $v_{\max }=3075$, 2965, 2823, 1725, 1605, 1582, 1570, 1500, 1495, 1455, 1245, 1025, 840, 832, 815, $750 \mathrm{~cm}^{-1} .{ }^{1} \mathrm{H}$ NMR (400 MHz, $\left.\mathrm{CDCl}_{3}\right): \delta$ $9.53(\mathrm{~d}, J=8.0 \mathrm{~Hz}, 1 \mathrm{H}), 8.21(\mathrm{~d}, J=7.4 \mathrm{~Hz}, 1 \mathrm{H}), 7.90$ (d, $J=7.9$ $\mathrm{Hz}, 1 \mathrm{H}), 7.81-7.68(\mathrm{~m}, 6 \mathrm{H}), 7.49$ (t, $J=7.4 \mathrm{~Hz}, 1 \mathrm{H}), 7.45$ (d, $J=$ $8.6 \mathrm{~Hz}, 2 \mathrm{H}), 7.15$ (d, $J=8.6 \mathrm{~Hz}, 2 \mathrm{H}), 3.98(\mathrm{~s}, 3 \mathrm{H}) .{ }^{13} \mathrm{C}$ NMR $(100$ $\mathrm{MHz}_{\mathrm{CDCl}}$ ): $\delta 191.5,162.2,160.8,149.7,148.0,144.1,137.5$, $135.5,134.9,132.1,131.6,131.5,129.6,128.1,128.0,127.6$, 126.2, 125.6, 125.4, 124.7, 124.1, 123.6, 121.8, 114.0, 55.8. Anal. Calcd for $\mathrm{C}_{27} \mathrm{H}_{17} \mathrm{NO}_{2}$ : C 83.72\%, H 4.39\%, N 3.61\%. Found: C 83.66\%, H 4.36\%, N 3.54\%.

7-(2-Methoxylphenyl)-8H-benzo[f]indeno-[1,2-b]quinoline8-one (4j). Yellow solid; mp: $269-270{ }^{\circ} \mathrm{C}$. IR (KBr): $v_{\max }=3070$, 2925, 2825, 1705, 1602, 1584, 1575, 1485, 1455, 1261, 1025, $845,812,753 \mathrm{~cm}^{-1} .{ }^{1} \mathrm{H}$ NMR (400 MHz, $\mathrm{CDCl}_{3}$ ): $\delta 9.55$ (d, $J=8.0$ $\mathrm{Hz}, 1 \mathrm{H}), 8.25$ (d, J = 7.4 Hz, 1H), 7.91 (d, J = 7.8 Hz, 1H), 7.79 (t, J $=8.0 \mathrm{~Hz}, 1 \mathrm{H}), 7.76-7.68(\mathrm{~m}, 4 \mathrm{H}), 7.59-7.55(\mathrm{~m}, 2 \mathrm{H}), 7.47(\mathrm{t}, J=$ $7.37 \mathrm{~Hz}, 1 \mathrm{H}$ ), 7.30 (d, J = 7.5 Hz, 1H), $7.20(\mathrm{t}, J=7.4 \mathrm{~Hz}, 1 \mathrm{H}), 7.17$ (d, $J=8.4 \mathrm{~Hz}, 1 \mathrm{H}), 3.74(\mathrm{~s}, 3 \mathrm{H}) \cdot{ }^{13} \mathrm{C} \mathrm{NMR}\left(100 \mathrm{MHz}, \mathrm{CDCl}_{3}\right): \delta$ 191.1, 162.2, 157.5, 149.7, 144.7, 144.4, 137.6, 135.4, 135.0, 132.2, 131.5, 131.1, 130.9, 129.4, 128.0, 127.9, 127.4, 126.2, 126.0, 124.8, 124.6, 124.1, 123.0, 121.8, 121.0, 111.7, 56.2. Anal. Calcd for $\mathrm{C}_{27} \mathrm{H}_{17} \mathrm{NO}_{2}$ : C $83.72 \%$, H 4.39\%, N 3.61\%. Found: C 83.62\%, H 4.35\%, N 3.52\%.

\section{Results and discussion}

To optimize the reaction conditions, we carried out the condensation reaction of 2-naphtylamine (1), 4-chlorobenzaldehyde (2b), and indane-1,3-dione (3) by varying the amount of $\mathrm{P}(4-\mathrm{VPH}) \mathrm{HSO}_{4}(5,10,20 \mathrm{mg})$, the reaction temperature (room temperature, $50{ }^{\circ} \mathrm{C}$, and reflux), and the solvent $\left(\mathrm{CH}_{2} \mathrm{Cl}_{2}\right.$, $\mathrm{CH}_{3} \mathrm{CN}, \mathrm{MeOH}, \mathrm{EtOH}$, and $\mathrm{H}_{2} \mathrm{O}$ ) (Scheme 1). The highest yield $(80 \%)$ was obtained in the presence of $20 \mathrm{mg} \mathrm{P}(4-\mathrm{VPH}) \mathrm{HSO}_{4}$ in EtOH at reflux for $2 \mathrm{~h}$.

We then used the optimized conditions to convert various aromatic aldehydes 2 (Scheme 1) into the corresponding 7-aryl-8H-benzo[f]indeno[1,2- $b]$ quinoline-8-one derivatives $\mathbf{4 a - 4 j}$ (Table 1). In all cases, the reactions proceeded readily to the desired products without the formation of any side products. This method was general and tolerated various substituents on the aromatic aldehydes (entries 1-10) , including electron-donating and electron-withdrawing substituents. The yields of benzo[f]indeno[1,2-b]quinolines were strongly af-

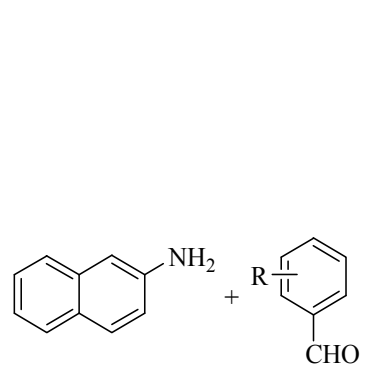

(1)

(2)

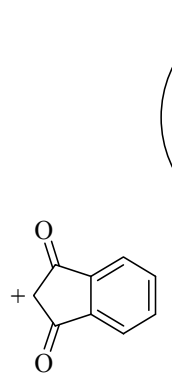

(3)

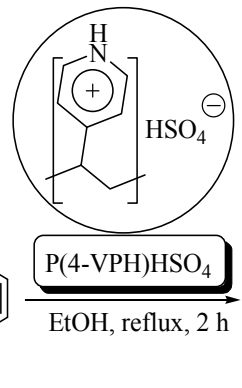

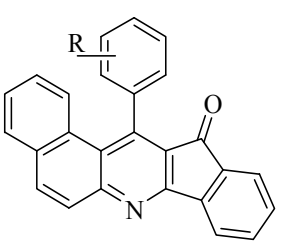

(4)

Scheme 1. Synthesis of 7-aryl-8H-benzo[f]indeno[2,1-b]quinoline-8-one derivatives in the presence of $\mathrm{P}(4-\mathrm{VPH}) \mathrm{HSO}_{4}$ in refluxing ethanol. 
fected by the electronic nature and the position of the substituent on the aromatic aldehydes. Aldehydes with electron-withdrawing substituents provided the desired benzo[f]indeno[1,2- $b]$ quinolines in good yields, whereas the yields were slightly lower for aldehydes with electron-donating substituents (entries 9 and 10). Aldehydes with ortho substituents gave slightly lower yields than those with para substituents (entries 2, 3, 9, and 10). Aliphatic aldehydes were not good substrates; the reactions did not progress, and TLC and GC-MS analysis of the reaction mixtures showed numerous products. It should be mentioned that when the model reaction was carried out for $2 \mathrm{~h}$ in the absence of catalyst under the optimized conditions, the yield of the product was low $(<30 \%)$.

The structures of the products were established from their analytical data: IR, ${ }^{1} \mathrm{H}$ NMR, ${ }^{13} \mathrm{C} \mathrm{NMR}$, and mass spectra, as well as elemental analysis. In the ${ }^{13} \mathrm{C}$ NMR spectra of all the products, the resonance for the carbonyl group appeared at $\delta=$ 190-193 ppm.

After the reaction, the catalyst could be isolated by filtration and recycled up to seven times without any significant loss of activity (Table 1, entry 2).

A possible mechanism for this reaction is shown in Scheme
2. Aldehydes $\mathbf{2}$ are activated by donation of a proton from $\mathrm{P}(4-\mathrm{VPH}) \mathrm{HSO}_{4}$ to the oxygen atom. Next, the carbonyl carbon is attacked by the nucleophilic indane-1,3-dione (3) to form intermediate I. Subsequent Michael addition of 2-naphthylamine (1) followed by cyclization and oxidation by air affords aromatized products 4 [103-107].

We observed no leakage of sulfuric acid from the matrix under the optimized reaction conditions. The catalyst showed an activity loss of $1 \%$ in the fourth use (in this run, a small amount of sulfuric acid leakage was detected in the reaction medium and wash solutions). However, after ten cycles of repeated use, the catalyst retained about $92 \%$ of its initial activity (data not shown); we attributed this result to the strong interactions between the pyridyl units of poly(4-vinylpyridine) and the acid sites. When the yield started to drop, the activity of the catalyst could be recovered by means of a simple activation procedure, and a high yield of 7-(4-chlorophenyl)-8H-benzo[f]indeno-[1,2- $b]$ quinoline-8-one was obtained in the presence of reactivated catalyst. The activity of the catalyst was not decreased, and the yield was $80 \%$ under the optimized reaction conditions.

Table 1

Synthesis of 7-aryl-8H-benzo[f]indeno[2,1-b]quinoline-8-one catalyzed by $\mathrm{P}(4-\mathrm{VPH}) \mathrm{HSO}_{4}$.

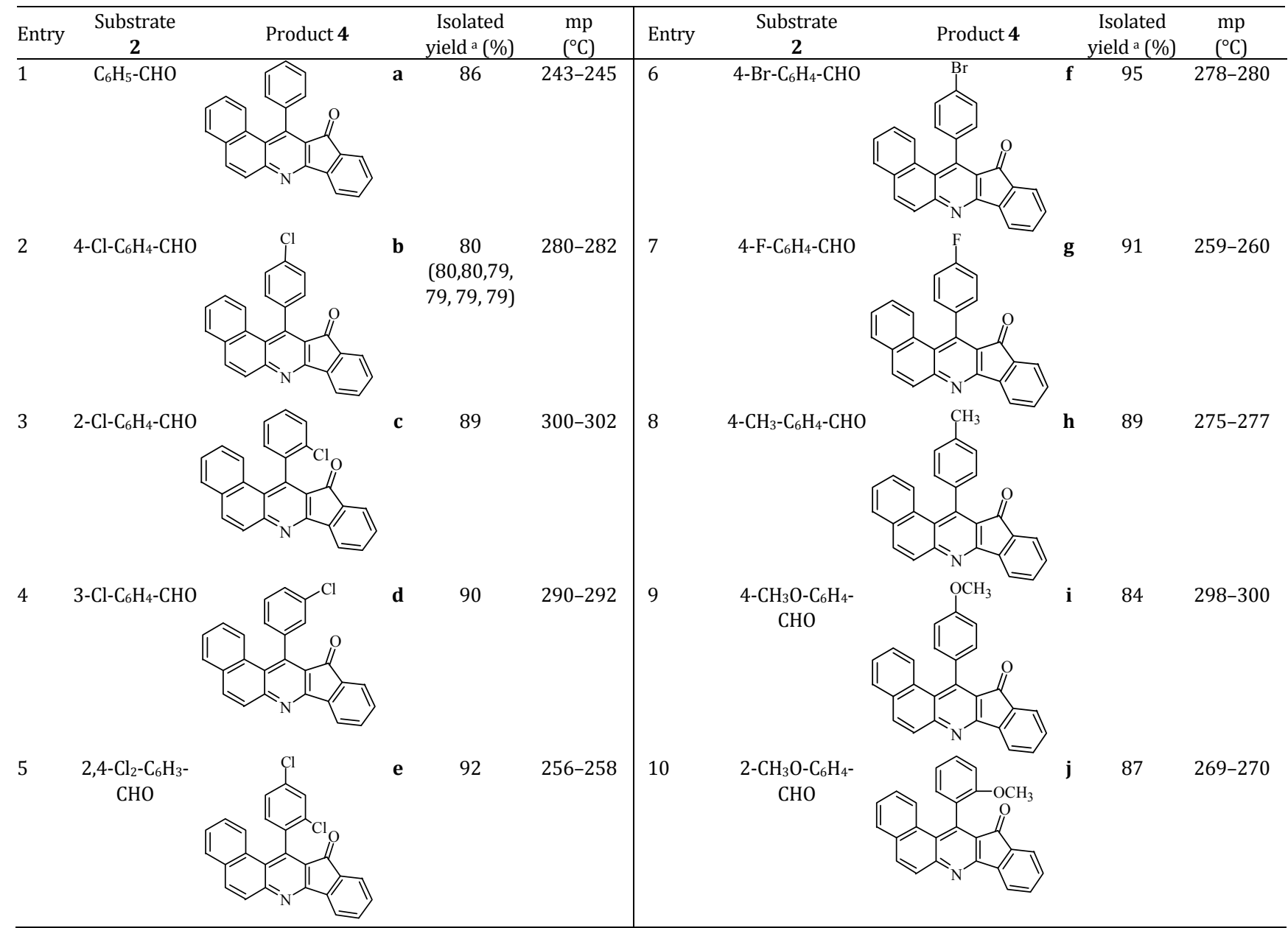

Reaction conditions: 2-naphthylamine $1.1 \mathrm{mmol}$, aromatic aldehyde $1 \mathrm{mmol}$, indane-1,3-dione $1 \mathrm{mmol}$, catalyst $20 \mathrm{mg}$, refluxed in ethanol $2 \mathrm{~h}$.

a Recycled solid acid catalyst. 


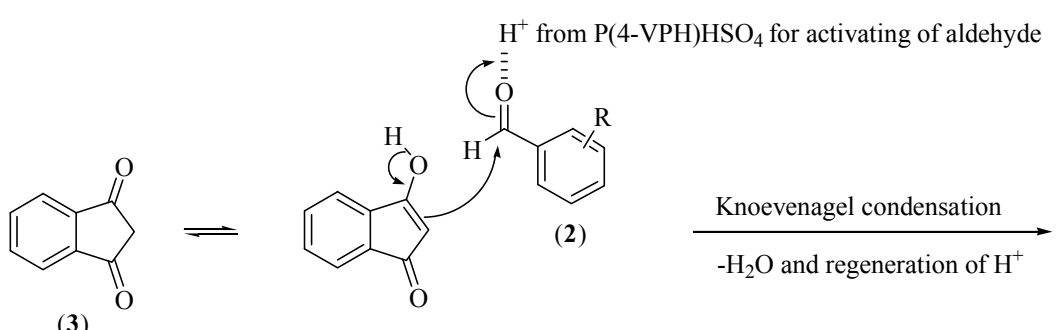

(3)<smiles></smiles><smiles>[R]c1ccccc1-c1c2c(nc3ccc4ccccc4c13)-c1ccccc1C2=O</smiles><smiles>[R][C]1CC=CC=C1C1C(=O)c2ccccc2-c2[nH]c3ccc4ccccc4c3c21</smiles>

Intramolecular condensation

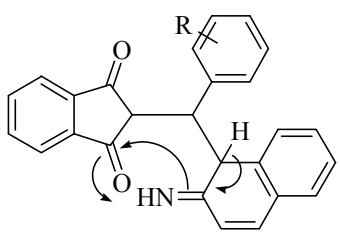

(4)

Scheme 2. A possible mechanism for the formation of 7-aryl- $8 H$-benzo[f]indeno[1,2-b]quinoline-8-ones in the presence of $\mathrm{P}(4-\mathrm{VP}) \mathrm{HSO}_{4}$ in refluxing ethanol.

\section{Conclusions}

We developed a novel, highly efficient method for the synthesis of 7-aryl-8H-benzo[f]indeno[1,2- $b]$ quinoline-8-one derivatives. The method is operationally simple and environmentally friendly, uses an inexpensive, readily available, and recyclable catalyst, and affords high to excellent yields of the products.

\section{References}

[1] Brock E D, Lewis D M, Yousaf T I, Harper H H. WO 9951688. 1999

[2] Beagley P, Blackie M A L, Chibale K, Clarkson C, Meijboom R, Moss J R, Smith P J, Su H. Dalton Trans, 2003: 3046

[3] Sawada Y, Kayakiri H, Abe Y, Mizutani T, Inamura N, Asano M, Hatori C, Aramori I, Oku T, Tanaka H.J Med Chem, 2004, 47: 2853

[4] Ma Z Z, Hano Y, Nomura T, Chen Y J. Bioorg Med Chem Lett, 2004, 14: 1193

[5] Denton T T, Zhang X D, Cashman J R. J Med Chem, 2005, 48: 224
[6] Fokialakis N, Magiatis P, Chinou L I, Mitaku S, Tillequin F. Chem Pharm Bull, 2002, 50: 413

[7] Fossa P, Mosti L, Menozzi G, Marzano C, Baccichetti F, Bordin F. Bioorg Med Chem, 2002, 10: 743

[8] Ryckebusch A, Deprez-Poulain R, Maes L, Debreu-Fontaine M A, Mouray E, Grellier P, Sergheraert C. J Med Chem, 2003, 46: 542

[9] Morgan L R, Jursic B S, Hooper C L, Neumann D M, Thangaraj K, LeBlanc B. Bioorg Med Chem Lett, 2002, 12: 3407

[10] Sakata G, Makino K, Karasawa Y. Heterocycles, 1988, 27: 2481

[11] Demeunynck M, Moucheron C, Mesmaeker A K D. Tetrahedron Lett, 2002, 43: 261

[12] Baraznenok I L, Nenajdenko V G, Balenkova E S. Eur J Org Chem, 1999: 937

[13] Ali M M, Tasneem K C, Rajanna K C, Prakash P K S. Synlett, 2001: 251

[14] Palacios F, de Retana A M 0, Oyarzabal J. Tetrahedron, 1999, 55: 5947

[15] Meshram H M, Reddy G S, Reddy M M, Yadav J S. Tetrahedron Lett, 1998, 39: 4103

[16] Cho C S, Kim B T, Kim T J, Shim S C. Chem Commun, 2001: 2576

[17] Crousse B, Begue J P, Bonnet-Delpon D. J Org Chem, 2000, 65: 5009

\section{Graphical Abstract}

Chin. J. Catal., 2014, 35: 474-480 doi: 10.1016/S1872-2067(12)60752-9

Three-component, one-pot synthesis of benzo[f]indenoquinoline derivatives catalyzed by poly(4-vinylpyridinium) hydrogen sulfate

Nader Ghaffari Khaligh*

Islamic Azad University, Iran; University of Guilan, Iran

One-pot condensation of 2-naphthylamine, aromatic aldehydes, and indane-1,3-dione was performed in refluxing ethanol in the presence of poly(4-vinylpyridinium) hydrogen sulfate as a catalyst. The catalyst could be recycled and reused four times without significant loss of activity.

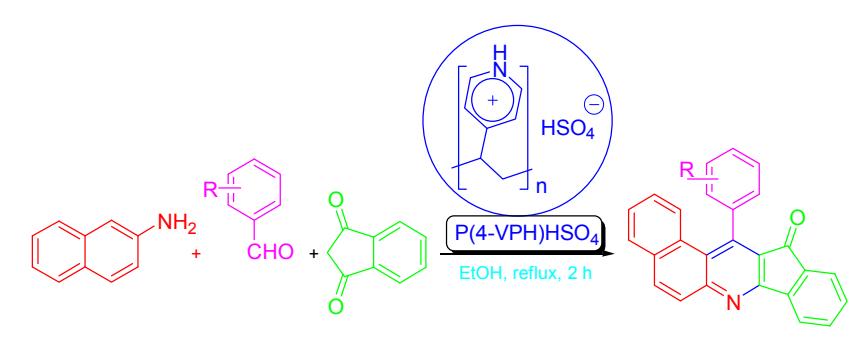


[18] Lin X F, Cui S L, Wang Y G. Tetrahedron Lett, 2006, 47: 4509

[19] Lin XF, Cui SL, Wang YG. Tetrahedron Lett, 2006, 47: 3127

[20] Skraup H. Chem Ber, 1880, 13: 2086

[21] Manske R H F, Kulka M. Org React, 1953, 7: 59

[22] Doebner O, Miller V W. Chem Ber, 1881, 14: 2812

[23] Conrad M, Limbach L. Chem Ber, 1887, 20: 944

[24] Combes A. Compt Rend, 1888, 106: 142

[25] Pfitzinger W.J Prakt Chem, 1886, 33: 100

[26] Povarov L S. Russ Chem Rev, 1967, 36: 656

[27] Kouznetsov V V. Tetrahedron, 2009, 65: 2721

[28] Haghighi M G, Rashidi M, Nabavizadeh S M, Jamali S, Puddephatt R J. Dalton Trans, 2010, 39: 11396

[29] Baratta W, Fanfoni L, Magnolia S, Siega K, Rigo P. Eur J Inorg Chem, 2010: 1419

[30] Baratta W, Ballico M, Baldino S, Chelucci G, Herdtweck E, Siega K, Magnolia S, Rigo P. Chem Eur J, 2008, 14: 9148

[31] Prema D, Wiznycia A V, Scott B M T, Hilborn J, Desper J, Levy C J. Dalton Trans, 2007: 4788

[32] Atatreh N, Stojkoski C, Smith P, Booker G W, Dive C, Frenkel A D, Freeman S, Bryce R A. Bioorg Med Chem Lett, 2008, 18: 1217

[33] Cappelli A, Giuliani G, Gallelli A, Valenti S, Anzini M, Mennuni L, Makovec F, Cupello A, Vomero S. Bioorg Med Chem, 2005, 13: 3455

[34] Murthy M, Pedemonte N, MacVinish L, Malietta L, Cuthbert A. Eur J Pharmacol, 2005, 516: 118

[35] Szkotak AJ, Murthy M, MacVinish L J, Duszyk M, Cuthbert A W. Br J Pharmacol, 2004, 142: 531

[36] Kerry M A, Boyd G W, Mackay S P, Meth-Cohn O, Platt L. J Chem Soc, Perkin Trans 1, 1999: 2315

[37] Cappelli A, Anzini M, Vomero S, Mennuni L, Makovec F, Doucet E, Hamon M, Bruni G, Romeo M R, Menziani M C, De Benedetti P G, Langer T. J Med Chem, 1998, 41: 728

[38] Tsai A S, Tauchert M E, Bergman R G, Ellman JA. J Am Chem Soc, 2011, 133: 1248

[39] Kakiuchi F, Kochi T, Mutsutani H, Kobayashi N, Urano S, Sato M, Nishiyama S, Tanabe T. J Am Chem Soc, 2009, 131: 11310

[40] Yu W Y, Sit W N, Lai K M, Zhou Z Y, Chan A S C. J Am Chem Soc, 2008, 130: 3304

[41] Dick A R, Hull K L, Sanford M S. J Am Chem Soc, 2004, 126: 2300

[42] Paritala H, Firestine S M. Bioorg Med Chem Lett, 2009, 19: 1584

[43] Patel D R, Patel K C. Fiber Polym, 2011, 12: 741

[44] Parekh N M, Maheria K C. Arch Appl Sci Res, 2011, 3: 359

[45] Sener I, Karci F, Ertan N, Kilic E. Dyes Pigm, 2006, 70: 143

[46] Karci F, Sener N, Yamac M, Sener I, Demircali A. Dyes Pigm, 2009, 80: 47

[47] Aramoto H, Obora Y, Ishii Y.J Org Chem, 2009, 74: 628

[48] Wang X S, Li Q Yao C S, Tu S J. Eur J Org Chem, 2008: 3513

[49] Kozlov N G, Gusak K N. Russ J Org Chem, 2008, 44: 1049

[50] Hewlins M J E, Salter R. Synthesis, 2007: 2157

[51] Li A H, Ahmed E, Chen X, Cox M, Crew A P, Dong H Q, Jin M Z, Ma L F, Panicker B, Siu K W, Steinig A G, Stolz K M, Tavares P A R, Volk B, Weng Q H, Werner D, Mulvihill M J. Org Biomol Chem, 2007, 5: 61

[52] Alajarin M, Vidal A, Ortin M M. Tetrahedron, 2005, 61: 7613

[53] Sangu K, Fuchibe K, Akiyama T. Org Lett, 2004, 6: 353

[54] Mahata P K, Venkatesh C, Kumar U K S, Ila H, Junjappa H. J Org Chem, 2003, 68: 3966

[55] Seeman P, Guan H C, Nobrega J, Jiwa D, Markstein R, Balk J H, Picetti R, Borrelli E van, Tol H H M. Synapse, 1997, 25: 137

[56] Elslager E F, Short F W, Tendick H F. J Heterocycl. Chem, 1968, 5: 599

[57] Bierer D E, Dubenko L G, Zhang P S, Lu Q, Imbach P A, Garofalo A
W, Phuan P W, Fort D M, Litvak J, Gerber R E, Sloan B, Luo J, Cooper R, Reaven G M. J Med Chem, 1998, 41: 2754

[58] Brooks J R, Berman D, Glitzer M S, Gordon L R, Primka R L, Reynolds G F, Rasmusson G H. Prostate, 1982, 3: 35

[59] Babu G, Perumal P T. Tetrahedron Lett, 1998, 39: 3225

[60] Yadav J S, Reddy B V S, Srinivas R, Madhuri Ch, Ramaligam T. Synlett, 2001: 240

[61] Yamato M, Takeuchi Y, Hashigaki K, Ikeda Y, Chang M R, Takeuchi K, Matsushima M, Tsuruo T, Tashiro T, Tsukagoshi S, Yamashita Y, Nakano H.J Med Chem, 1989, 32: 1295

[62] Lu X L, Petersen J L, Wang K K. Org Lett, 2003, 5: 3277

[63] Rampa A, Bisi A, Belluti F, Gobbi S, Valenti P, Andrisano V, Cavrini V, Cavalli A, Recanatini M. Bioorg Med Chem, 2000, 8: 497

[64] Deady L W, Desneves J, Kaye A J, Finlay G J, Baguley B C, Denny W A. Bioorg Med Chem, 2000, 8: 977

[65] Deady L W, Desneves J, Kaye A J, Finlay G J, Baguley B C, Denny W A. Bioorg Med Chem, 2001, 9: 445

[66] Bu X Y, Deady L W. Synth Commun, 1999, 29: 4223

[67] Schmittel M, Strittmatter M, Vollmann K, Kiau S. Tetrahedron Lett, 1996, 37: 999

[68] Cho C S, Kim B T, Choi H J, Kim T J, Shim S C. Tetrahedron, 2003, 59: 7997

[69] Sunami T, Nishio K, Kanzawa F, Fukuoka K, Kudoh S, Yoshikawa J, Saijo N. Cancer Chemother Pharmacol, 1999, 43: 394

[70] Atwell G J, Rewcastle G W, Baguley B C, Denny W A. J Med Chem, 1987, 30: 664

[71] Spicer J A, Gamage S A, Atwell G J, Finlay G J, Baguley B C, Denny W A. J Med Chem, 1997, 40: 1919

[72] Kohlhagen G, Paull K D, Cushman M, Nagafuji P, Pommier Y. Mol Pharmacol, 1998, 54: 50

[73] Antony S, Jayaraman M, Laco G, Kohlhagen G, Kohn K W, Cushman M, Pommier Y. Cancer Res, 2003, 63: 7428

[74] Claret P A, Osborne A G: In: Jones G Ed. The Chemistry of Heterocyclic Compounds (Vol. 32, Part 2). Quinolines: John Wiley \& Sons, 1982. 31

[75] Sawada Y, Kayakiri H, Abe Y, Imai K, Mizutani T, Inamura N, A sano M, Aramori I, Hatori C, Katayama A, Oku T, Tanaka H. J Med Chem, 2004, 47: 1617

[76] Groisy-Delcey M, Groisy A, Carrez D, Huel C, Chiaroni A, Ducrot P, Bisagni E, Jin L, Leclercq G. Bioorg Med Chem, 2000, 8: 2629

[77] Cheng C H, Chen R M, Huang C W, Yang C C. US 20050025995A1. 2005

[78] Kim J I, Shin I S, Kim H, Lee J K. J Am Chem Soc, 2005, 127: 1614

[79] Thompson M E, Ma B W, Djurovich P. US 20050164031A1. 2005

[80] Sun Y, Sun J, Yan C G. Beilstein J Org Chem, 2013, 9: 8

[81] Agra A K, Jenekhe S A. Macromolecules, 1991, 24: 6806

[82] Zhang X J, Shetty A S, Jenekhe S A. Macromolecules, 1999, 32: 7422

[83] Jenekhe S A, Lu L D, Alam M M. Macromolecules, 2001, 34: 7315

[84] Khaligh N G. Tetrahedron Lett, 2012, 53: 1637

[85] Mamaghani M, Larghani T H. J Chem Res, 2012, 36: 235

[86] Wang X S, Zhang M M, Zeng Z S, Shi D Q Tu S J, Wei X Y, Zong Z M. J Heterocycl Chem, 2006, 43: 989

[87] Tu S J, Zhang Y, Zhang J Y, Jiang B, Jia R H, Zhang J P, Ji S J. Synlett, 2006: 2785

[88] Tu S J, Wu S S, Yan S, Hao W J, Zhang X H, Cao X D, Han Z G, Jiang B, Shi F, Xia M, Zhou J F. J Comb Chem, 2009, 11: 239

[89] Heravi M M, Hosseini T, Derikvand F, Beheshtiha S Y S, Bamoharram F F. Synth Commun, 2010, 40: 2402

[90] Tanabe K, Hölderich W F. Appl Catal A, 1999, 181: 399

[91] Ghorbani-Choghamarani A, Zolfigol M A, Hajjami M, Sardari S. Synth Commun, 2013, 43: 52

[92] Li M, Chen D Y, Zhu X F. Chin J Catal (催化学报), 2013, 34: 1674 
[93] Rahi T, Baghernejad M, Niknam K. Chin J Catal (催化学报), 2012, 33: 1095

[94] Cole-Hamilton D J. Science, 2003, 299: 1702

[95] Chakrabarti A, Sharma M M. React Polym, 1993, 20: 1

[96] Riego J M, Sedin Z, Zaldivar J M, Marziano N C, Tortato C. Tetrahedron Lett, 1996, 37: 513

[97] Turro N J. Tetrahedron, 1987, 43: 1589

[98] Zolfigol M A, Chehardoli G, Dehghanian M, Niknam K, Shirini F, Khoramabadi-Zad A. J Chin Chem Soc (Taipei), 2008, 55: 885

[99] Weber U S, Steffen B, Siegers C P. Res Commun Mol Pathol Pharmacol, 1998, 99: 193
[100] Li Y O. Petrochem Technol, 1981, 54: 309

[101] Patil A D, Freyer A J, Eggleston D S, Haltiwanger R C, Bean M F, Taylor P B, Caranfa M J, Breen A L, Bartus H R, Johnson R K, Hertzberg R P, Westley J W. J Med Chem, 1993, 36: 4131

[102] Khaligh N G, Shirini F. J Mol Catal A, 2011, 348: 20

[103] Wang X S, Zhou J, Yang K, Li Y L. Tetrahedron Lett, 2011, 52: 612

[104] Tapaswi P K, Mukhopadhyay C. Arkivoc, 2011: 276

[105] Bhargava G, Mohan C, Mahajan M P. Tetrahedron, 2008, 64: 3017

[106] Shi D Q, Ni S N, Yang F, Shi J W, Dou G L, Li X Y, Wang X S, Ji S J. J Heterocycl Chem, 2008, 45: 693

[107] Shi D Q, Yang F, Ni S N. J Heterocycl Chem, 2009, 46: 469 